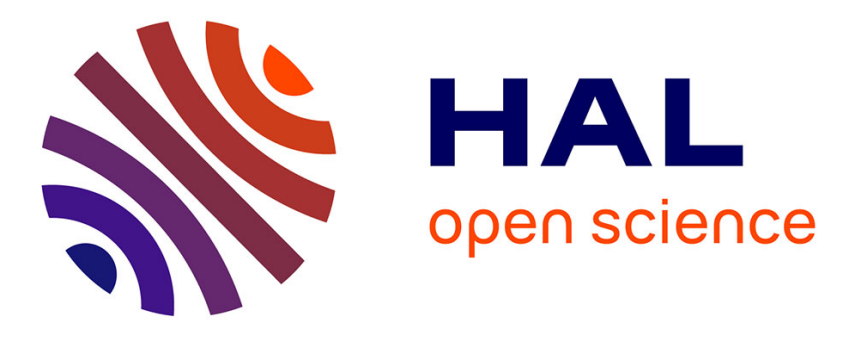

\title{
Some Control and Observation Issues in Cellular Automata
}

Samira El Yacoubi, Théo Plénet, Sara Dridi, Franco Bagnoli, Laurent Lefèvre, Clément Raïevsky

\section{- To cite this version:}

Samira El Yacoubi, Théo Plénet, Sara Dridi, Franco Bagnoli, Laurent Lefèvre, et al.. Some Control and Observation Issues in Cellular Automata. Complex Systems , 2021, 30 (3), pp.391-413. 10.25088/ComplexSystems.30.3.391 . hal-03357832

\section{HAL Id: hal-03357832 https://hal.science/hal-03357832}

Submitted on 29 Sep 2021

HAL is a multi-disciplinary open access archive for the deposit and dissemination of scientific research documents, whether they are published or not. The documents may come from teaching and research institutions in France or abroad, or from public or private research centers.
L'archive ouverte pluridisciplinaire HAL, est destinée au dépôt et à la diffusion de documents scientifiques de niveau recherche, publiés ou non, émanant des établissements d'enseignement et de recherche français ou étrangers, des laboratoires publics ou privés. 


\title{
Some Control and Observation issues in Cellular Automata
}

\author{
Samira El Yacoubi and Théo Plénet \\ IMAGES-ESPACE-DEV, Univ. Perpignan Via Domitia, Perpignan, France \\ ESPACE-DEV, Univ Montpellier, IRD, Montpellier France \\ Sara Dridi \\ Laboratory of Applied Mathematics-LaMA, University of Setif, Setif, Algeria \\ Franco Bagnoli \\ Dept. Physics and Astronomy and CSDC, University of Florence \\ via G. Sansone 1, 50019 Sesto Fiorentino (FI), Italy also INFN, sez. Firenze \\ Laurent Lefèvre and Clément Raïevsky \\ Univ. Grenoble Alpes, Grenoble INF LCIS, 26000 Valence, France
}

\begin{abstract}
This review article focuses on studying problems of observability and controllability of Cellular Automata considered in the context of control theory, an important feature of which is the adoption of a state space model. Our work first consists in generalizing the obtained results to systems described by cellular automata considered as the discrete counterpart of PDEs, and in exploring possible approaches to prove controllability and observability. After having introduced the notion of control and observation in cellular automata models, in a similar way to the case of discrete-time distributed parameter systems, we investigate these key concepts of control theory in the case of complex systems. For the controllability issue, the Boolean class is particularly studied and applied to the regional case while the observability is approached in the general case and related to the reconstructibility problem for linear or nonlinear cellular automata.
\end{abstract}

Keywords: cellular automata; control systems; controllability; observability

\section{Introduction}

Controllability and observability are among the most prominent and main considered issues in control theory. They were introduced by Kalman and well studied during the second half of the last century. A wide variety of works related to controllability and observability of distributed parameters systems (DPS) has been achieved 13. The study of these notions on DPS via the structure of actuators (inputs) and sensors (outputs) was the subject of an intense research activ-

*Institute of Engineering Univ. Grenoble Alpes

Complex Systems, Volume (year) 1-1+; year Complex Systems Publications, Inc. 
ity [13, 12. The traditional and most used models in these studies are based on a set of partial differential equations (PDEs) for the description of system input-output dynamics. Whereas controllability concerns the ability to steer the processes so as to bring them towards desired profiles through specific actions, observability deals with the ability of reconstructing the initial system state, taking into account a sufficient knowledge of the system dynamics based on specified output measurements. These two major concepts have already been studied for continuous systems described by PDEs as reported in the literature [13, 4, 40]. In the case of deterministic linear systems analysis, the so-called Kalman condition [20, 21] is essential and has been widely used to obtain the main characterization results regarding the choice of actuator/sensor structures, locations, number, and types (mobile or fixed). See for example [13, 14] and the references therein.

The aim of this paper is to summarize some recent advances on controllability and observability of systems described by cellular automata, considered as the discrete counterpart of PDEs, and explore other suitable approaches to prove controllability and observability for such systems. Cellular automata (CA) are widely used mathematical models for studying dynamical properties of discrete systems and constitute very promising tools for describing complex natural systems in terms of local interactions [3, 33, 32].

Cellular automata are the simplest models of spatially extended systems which may provide a good description for complex phenomena. They are discrete dynamical systems where space, time, as well as states related to physical quantities, are all discrete. Evolution is governed by a set of simple local and microscopic transition rules that may exhibit a complex behaviour. In the macroscopic limit (i.e., after space and time coarse graining), CA can reproduce the same phenomena usually modelled with PDEs. A wide range of applications in biology, chemistry, physics, and ecology was successfully developed using the CA paradigm as reported in the large dedicated literature [16, 34, 25].

Since CA models were so far considered as autonomous systems, the idea was first to introduce the notion of control and observation in these models, in a similar way is was previously done for discretetime distributed parameter systems, in order to be able to study some concepts of control theory related to inputs and outputs [36]. We then started to study the two key and most popular concepts of control theory, namely controllability and observability, in the case of complex systems modelled by CA.

For the controllability problem, we focused on a particular case of the so-called regional controllability. In the context of distributed parameter systems, the term regional has been used to refer to control problems in which the desired state is only defined, and may be reachable, on some portion of the domain. In many physical problems the

Complex Systems, Volume (year) 1-1+ 
regional controllability is naturally chosen in order to shape a natural phenomenon just in a sub region of the whole domain. The case of Boolean CA has been particularly examined to investigate the boundary regional controllability [9]. It consists in considering objective functions defined on a sub-region of the domain and exerting control actions on the boundary of the target region. This problem has been dealt with several tools: namely Kalman theorem, Markov Chains, graph theory 10, 11. The extension to non-linear CA has also been studied in these works.

For the problem of observability, we assume that the studied system is autonomous and we apply the tools mentioned above to prove observability as a dual notion of controllability. Where, in controllability analysis, the state of the system has to be steered to a desired value using an unknown input signal with minimal energy, in the case of observability we seek to observe through a given output signal the maximum energy of the unknown state of the system. The first results were obtained for affine CA and a rank condition for observability was proved in 28]. This condition is reminiscent of deterministic linear systems theory. Several criteria to assess the observability and the reconstructibility of CA were formulated according to the choice of sensor structures, locations, and types (mobile or fixed). Some examples were given to illustrate the theoretic results. The non-linear case as well as the probabilistic case are currently under investigation.

\section{Controllability of Cellular Automata}

\subsection{Introduction to Distributed Parameters Systems}

Several phenomena exhibit spatially distributed behaviour and are distributed parameter systems (DPS) where the state variables depend on time and space and describes the system's behaviour in terms of inputs and outputs that also depend on space and time.

Although DPS are more common in industry, their applications have been expanded to include biological, ecological, or economic systems and are becoming extremely related to the study of complex systems. Depending on the application, various representations of DPS can be considered with different inputs and outputs structures. The usual form they take is based on a state-space representation described as a set of partial differential equations (PDEs) that provide detailed descriptions of the internal behaviour of the system.

The mathematical description of a DPS is usually given by three operators: $A$ describing the dynamics, $B$ and $C$ determining how the controls act on the system and how it is observed. 


$$
\left\{\begin{array}{l}
z^{\prime}(t)=A z(t)+B u(t) ; 0<t<T \\
z(0)=z_{0} \in D(A)
\end{array}\right.
$$

and augmented by the output equation:

$$
y(t)=C z(t)
$$

Two key concepts for analysing such systems are controllability and observability that are studied through operators $B$ and $C$. The controllability concerns the ability to steer the system from any initial state to any desired state by acting on inputs that are involved in the operator $B$. Whereas, the observability deals with the capability to reconstruct the initial state of the system, taking into account sufficient knowledge of the system's dynamics through certain output measurements according to the operator $C$.

The problem of controllability of DPS has been widely studied in recent years [13. Various types of controllability have been considered for DPS: exact, weak or regional controllability [26, 22. The regional case was introduced by Zerrik et al [40], as a special case of output controllability 26, 30. It consists in achieving an objective only on a region $\omega$ of the spatial domain on which the governing partial differential system is considered. This regional idea appears naturally in many real-world dynamical systems, when studying a natural phenomenon only in a specific area. This concept has been widely developed and interesting results have been proved, in particular, the possibility to reach a state on an internal subregion or on a part of the boundary of the domain when some specific actions are exerted on the system, in its domain interior or on its boundaries. Cellular automata (CA) models are particularly suitable for simulating natural phenomena that are usually highly non-linear and better described in terms of discrete units rather than by means of partial differential equations (PDEs) 23, 5, 8, 16. CA approach has been recently promoted for the study of control problems on spatially extended systems for which the classical approaches cannot be used. The addressed question: Can we consider CA as a possible alternative to DPS for modelling and analysis of spatially extended system?

\section{2.2 Discrete-time DPS statement by means of CA formalism}

Cellular Automata are spatially extended systems that are widely used for modelling various problems ranging from physics to biology, engineering, medicine, ecology, and economics. An ultimate understanding of such systems gives one the ability to control them in order to achieve desired behaviour.

Complex Systems, Volume (year) 1-1+ 
Definition 1. A CA is classically defined by a quadruple $\mathcal{A}=(\mathcal{L}, \mathcal{S}, \mathcal{N}, f)$ where

- $\mathcal{L}$ is a $d$-dimensional lattice of cells $c$ which are arranged depending on space dimension and cell shape. In the infinite case, $\mathcal{L}=\mathbb{Z}^{d}$.

- $\mathcal{S}$ denotes a discrete state set. It's a finite commutative ring given by $\mathcal{S}=\{0,1, \cdots, k-1\}$ in which the usual operations use modular arithmetic.

- $\mathcal{N}$ is a mapping which defines the cell's neighbourhood.

The neighbourhood is usually given by:

$$
\begin{aligned}
\mathcal{N}: & \mathcal{L} \\
c & \longrightarrow \mathcal{L}^{n} \\
& \longrightarrow \mathcal{N}(c)=\left\{c^{\prime} \in \mathcal{L} \mid\left\|c^{\prime}-c\right\|_{i} \leq r\right\}
\end{aligned}
$$

where $\|c\|_{i}, i \in\{1, \infty\}$ indicates the sum and the maximum respectively, of the absolute value of the components of cell $c$ (For $d=2$, $\left|\left\|c^{\prime}-c\right\|_{1}=\right| c_{i}^{\prime}-c_{i}|+| c_{j}^{\prime}-c_{j} \mid$ and $\left.\mid\left\|c^{\prime}-c\right\|_{\infty}=\max \left(\left|c_{i}^{\prime}-c_{i}\right|,\left|c_{j}^{\prime}-c_{j}\right|\right)\right)$.

- $f$ is a transition function which can be defined by :

$$
\begin{aligned}
& f: \mathcal{S}^{n} \longrightarrow \mathcal{S} \\
& s_{t}(\mathcal{N}(c)) \quad \longrightarrow \quad s_{t+1}(c)=f\left(s_{t}(\mathcal{N}(c))\right.
\end{aligned}
$$

where $s_{t}(c)$ designates the $c$ cell state at time $t$ and $s_{t}(\mathcal{N}(c))=\left\{s_{t}\left(c^{\prime}\right), c^{\prime} \in\right.$ $\mathcal{N}(c)\}$ is the state of the neighbourhood.

In the linear case, $f\left(x_{1}, \ldots, x_{n}\right)=\sum_{i=1}^{n} a_{i} x_{i}$, where the sum is taken modulo $k$. In the affine case, $f\left(x_{1}, \ldots, x_{n}\right)=\sum_{i=1}^{N} a_{i} x_{i}+c$.

We can have also cases in which the function $f$ is linear only for a certain set of variables. As we shall see, for control purposes the case of peripherally linear CA is particularly interesting, for which

$$
f\left(x_{1}, \ldots, x_{n}\right)=a_{1} x_{1}+h\left(x_{2}, \ldots, x_{n-1}\right)+a_{n} x_{n},
$$

where $h$ is an arbitrary function (linear or nonlinear).

In order to consider CA in the context of DPS, a description in the form of a state equation is necessary.

\subsubsection{New state equation}

Consider the case $\mathcal{L}=\mathbb{Z}^{d},(d \geq 1)$ and introduce a metric over $X=\mathcal{S}^{\mathbb{Z}^{d}}$ as :

$$
d_{\delta}(x, y)=\sum_{c \in \mathbb{Z}^{d}} \frac{\delta(x(c), y(c))}{2^{\|c\|_{\infty}}}
$$

where $\delta: \mathcal{S} \times \mathcal{S} \rightarrow\{0,1\}$ is defined by :

$$
\begin{aligned}
\delta(i, j)= \begin{cases}0 & \text { if } \quad i=j \\
1 & \text { if } \quad i \neq j\end{cases} \\
\text { Complex Systems, Volume (year) 1-1+ }
\end{aligned}
$$


The set $X=\mathcal{S}^{\mathcal{L}}$ equipped with the distance $d_{\delta}$ is a compact metric space and the global dynamics $F$

$$
\begin{aligned}
F: X & \rightarrow X \\
s & \rightarrow F(s)
\end{aligned}
$$

is continuous according to the topology induced by $d_{\delta}$.

Proposition 1. 36

- The compact configurations set $X$ defines the state space of the autonomous CA.

- The sequence of continuous global maps $F^{i}$ defined as the $i^{\text {th }}$ iteration under $F$, plays the same role than the semi-group, usually denoted by $\left(\Phi_{t}\right)$ generated by the operator $A$.

In a way similar to discrete-time DPS, the evolution of an autonomous CA starting from a given initial configuration $s_{0}$ can be defined in terms of the global dynamics by the state equation:

$$
\left\{\begin{array}{lll}
s_{t+1} & =F s_{t} \\
s_{0} & \in
\end{array}\right.
$$

In the linear case, the operator $F$ is simply a circulant band matrix $J$ (of width $n$ ),

$$
s_{t+1}=J s_{t},
$$

where the matrix sum-product is taken modulo $k$. In the affine case,

$$
s_{t+1}=J s_{t}+C .
$$

\subsubsection{Control and observation in cellular automata}

The CA model will be completed by control and measurement functions. For the control aspects, it is done via inputs (actuators) which have a spatial structure (number, spatial location and distribution).

Let us consider the following general hypothesis.

- $\mathcal{L}=\mathbb{Z}^{d}$ is a cellular domain whose elements $c=\left(i_{1}, \ldots, i_{d}\right)$,

- $I_{T}=\{0,1, \cdots, T\}$ is a discrete time horizon,

- $\mathcal{L}_{p}$ is a sub-domain which defines the region of the lattice $\mathcal{L}$ where the CA is excited. It contains $p$ cells which may be connected or not.

- The control operator $G$ which defines the way the control excites the CA through the cells of $\mathcal{L}_{p}$, is given by:

$$
\begin{aligned}
G: \mathcal{U} & \longrightarrow \mathcal{S}^{\mathbb{Z}^{d}} \\
u & \longrightarrow G u
\end{aligned}
$$

Complex Systems, Volume (year) 1-1+ 
where $\mathcal{U}=\ell^{2}\left(\mathcal{L}_{p}, \mathbb{R}\right)=\left\{u: \mathcal{L}_{p} \longrightarrow \mathbb{R} \mid \sum_{c \in \mathcal{L}_{p}} u^{2}(c)<\infty\right\}$ is the control space.

$G$ is an operator that transforms the physical actions $u$ to a realization $G u$ in the state space. Its action on cell $c$ is denoted as $g_{c}(u)$.

- The CA is then considered as a controlled system, defined by the local transition function:

$$
s_{t+1}(c)=f\left(s_{t}(\mathcal{N}(c))\right)+g_{c}\left(u_{t}\right)
$$

where again the sum is taken modulo $k$. Considering for simplicity the Boolean case, this means that where $g_{c}(u)$ is one, the actions of the $f_{c}$ is reversed, and where $g_{c}(u)$ is zero, it is not modified.

- The corresponding state equation is:

$$
\begin{cases}s_{t+1}= & F s_{t}+G u_{t}, t \in I_{T} \\ s_{0} & \in X\end{cases}
$$

- The observation problem can be considered by duality where an observation space and a global observation operator have to be defined.

For $I_{m}=\{0,1, \ldots, T m\}$ and $\mathcal{O}=\ell^{2}\left(\mathcal{L}_{q}, \mathbb{R}\right)$, the observation space consisting of all bounded measurements made in $\mathcal{L}_{q} \subset \mathcal{L}$ and given by a measurement variable (output) denoted by $\theta: I_{m} \longrightarrow \mathcal{O}$ that defines the measurement at time $t$. The global observation operator $H$ defined by:

$$
\begin{aligned}
H: \mathcal{S}^{\mathbb{Z}^{d}} & \longrightarrow \mathcal{O} \\
s & \longrightarrow
\end{aligned}
$$

associates a measurement to each configuration s.

- This leads to a complete description of CA in terms of inputs and outputs where the state equation is augmented with

$$
\theta_{t}=H s_{t}, t \in I_{m}
$$

and then defines the so-called distributed CA.

- The obtained CA statement is very close to the usual discrete-time distributed parameter systems formulation augmented by the output function.

The problem that we want to address here is that of forcing the appearance of a given pattern inside a region by imposing a suitable set of values to some specific sites that could be in the lattice or in its boundary.

The idea is to explore different formalisms and approaches, some of which are specific to PDEs, that can prove the regional controllability of CA, focusing on Boolean CA. 


\section{2.3 Regional controllability of CA: Kalman condition}

Let's recall the classical Kalman rank condition [20] as stated for the finite dimensional systems:

Definition 2. The controllability matrix related to the system (1) is a matrix of dimension $n \times n m$ defined by:

$$
M_{c}=\left[B, A B, \cdots, A^{n-1} B\right]
$$

The determination of the matrix gives an information whether the system is controllable or not. We have the following theorem (Kalman condition):

Theorem 1. The system (1) is controllable if and only if the controllability matrix is of full rank, in other words:

$$
\operatorname{rank}\left(M_{c}\right)=n
$$

This was generalised to $\mathrm{CA}$ and allowed to prove the regional controllability.

Definition 3. Let's consider:

- A CA defined on a discrete lattice $\mathcal{L}$ with state set $\mathcal{S}$ and described by a transition function $f$.

- $\omega \subset \mathcal{L}$

- $s_{\omega}$ the restriction to $\omega$ of the CA configuration $s$.

- $\mathcal{S}^{\omega}=\{s: \omega \rightarrow \mathcal{S}\}$

The $\mathrm{CA}$ is said to be regionally controllable if for a given $s_{d} \in \mathcal{S}^{\omega}$ there exists a control sequence $u=\left(u_{0}, \cdots, u_{T-1}\right)$ with $u_{i} \in \mathcal{U}$ such that:

$$
s_{T}=s_{d} \quad \text { on } \omega
$$

where $s_{T}$ is the final configuration at time $T$ and $\mathcal{U}$ is the control space.

\subsubsection{Special case of Boolean CA}

Let us consider a Boolean $\mathrm{CA}$ defined on a lattice $\mathcal{L}$ that is assumed to be finite and composed of $N$ interior cells and 2 boundary cells where the actions will be exerted, denoted by $c_{l}$ and $c_{r}$ for the left and right boundary cells, respectively. We are interested in finding the suitable sequences of controls acting on the boundary of the lattice, $\left(u_{l}^{0}, u_{l}^{1}, \cdots, u_{l}^{T-1}\right)$ and $\left(u_{r}^{0}, u_{l}^{r}, \cdots, u_{r}^{T-1}\right)$, so as to steer the system from a given initial state $s_{0}$ to a desired configuration $s_{d}$ on the subregion $\omega$ at a given time $T$, such that: $s_{T}\left(c_{i}\right)=s_{d}\left(c_{i}\right), \forall c_{i} \in \omega$. The 
desired configuration $s_{d}$ is assumed to be reachable in the evolution of the CA rule.

In the following, to make notation more clear, we shall indicate with the symbol symbol $\oplus$ the sum modulo two.

The state equation for linear Boolean CA is

$$
\left\{\begin{array}{l}
s_{t+1}=J s_{t} \oplus G u_{t} ; 0<t<T \\
s_{0} \in X
\end{array}\right.
$$

where

- $J$ is the circulant band matrix each line of which contains the coefficients $a_{1}, \ldots, a_{n}$ in the positions corresponding to the neighbourhood $\mathcal{N}$.

- $G$ is a $(n \times 2)$ matrix that represents the control operator,

- $u_{t}=\left[u_{l}^{t}, u_{r}^{t}\right]$ is the control matrix at time $t$ consisting of a 2-component vector in this particular case.

Theorem 2. Kalman condition for controllability.

A 1D linear Cellular Automata is regionally controllable via boundary actions iff:

$$
\operatorname{Rank}\left(M_{c}\right)=\operatorname{Rank}\left(B \quad J B \quad J^{2} B \ldots J^{T-1} B\right)=T=N-1
$$

Where $T$ is the time horizon, $N$ is the size of CA lattice and $J$ is the Jacobian matrix.

Proof. Let $s_{0}$ be the initial configuration of $\mathcal{A}$. Assume that the CA is regional controllable in $\omega_{1}$ by acting on $c_{l}$ such as $\omega_{1} \subset \omega$ and $\omega_{1}=\omega-\left\{c_{l}\right\}$, then it exists a sequence of control $\left\{u_{l}^{t}, u_{r}^{t}\right\}$ such as $t=0, \ldots T-1$. By using equation

$$
s^{t+1}=J s^{t} \oplus B u^{t}
$$

It follows that

$$
\begin{aligned}
& s^{1}=J s^{0} \oplus B u_{l}^{0} \\
& s^{2}=J s^{1} \oplus B u_{l}^{1} \\
& s^{2}=J\left(J s^{0} \oplus B u_{l}^{0}\right) \oplus B u_{l}^{1} \\
& s^{2}=J^{2} s^{0} \oplus J B u_{l}^{0} \oplus B u_{l}^{1} \\
& s^{3}=J s^{2} \oplus B u_{l}^{2} \\
& s^{3}=J\left(J^{2} s^{0} \oplus J B u_{l}^{0} \oplus B u_{l}^{1}\right) \oplus B u_{l}^{2} \\
& s^{3}=J^{3} s^{0} \oplus J^{2} B u_{l}^{0} \oplus J B u_{l}^{1} \oplus B u_{l}^{2}
\end{aligned}
$$

Therefore:

$$
\begin{array}{r}
s^{T}=J^{T} s^{0}+\left(\begin{array}{lll}
B & J B & J^{2} B \ldots J^{T-1} B
\end{array}\right)\left(\begin{array}{ll}
u_{l}^{T-1} & u_{l}^{T-2} \ldots u_{l}^{0}
\end{array}\right)^{t r} \\
\text { Complex Systems, Volume (year) } 1-1+
\end{array}
$$


We define the controllability matrix $M_{c}$ :

$$
\left(\begin{array}{lll}
B & J B & J^{2} B \ldots J^{T-1} B
\end{array}\right)
$$

We get the regional controllability when

$$
\operatorname{Rank}\left(M_{c}\right)=T
$$

For peripheral linear CA, we know also that for being able to change any state of region $\omega_{1}=\left\{c_{1}, c_{2}, \ldots, c_{N}\right\}$ from one boundary $c_{l}$ (if the CA is left-linear) the time $T$ should equal $N-1[2]$, where $N$ is the size of CA. Hence, we can get that:

$$
\operatorname{Rank}\left(M_{c}\right)=T=N-1
$$

Now suppose, $\operatorname{Rank}\left(M_{c}\right)=T=N-1$ where $\operatorname{Rank}\left(M_{c}\right)=\operatorname{dim}\left(\operatorname{Image}\left(M_{c}\right)\right)$ then for each initial configuration $s_{0}$ one can associates a desired configuration $s_{d}$. Hence the proof.

\section{Remark}

It is difficult to develop algorithms to decide whether a generic rule is controllable. But for linear rules or peripherally linear (that depend linearly on a peripheral site) this can be done iteratively: the least external site can control a site in the target region, flipping its value if wrong. And then this can be repeated for all sites to be controlled.

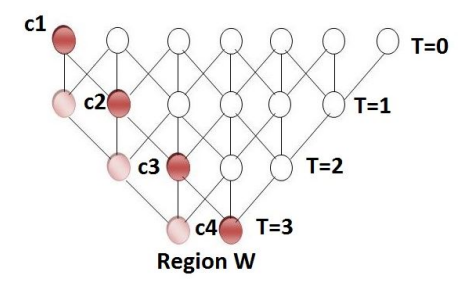

Example 1. Consider the example of the ECA rule 150. We suppose that we act only on the left boundary cell of a region $\omega=\left\{c_{10}, \ldots, c_{30}\right\}$ and show that there exists a sequence of controls $u=\left\{u_{c_{l}}^{0}, \ldots, u_{c_{l}}^{T-1}\right\}$ that steers the system in $\omega$ from the initial configuration to a desired one constituted of ones, $s_{d}=(1, \cdots, 1)$ at time $T=39$. In the following figures, we illustrate the evolution of CA rule 150 with (figure (a)) and without (figure (b)) the application of these controls. 


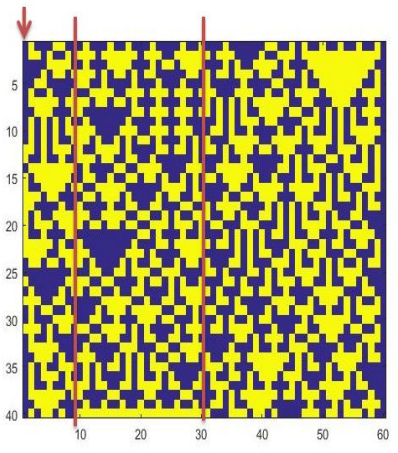

(a)

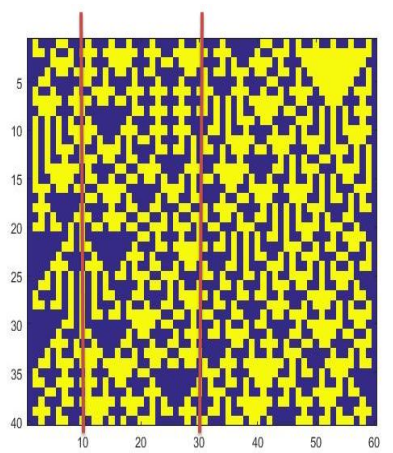

(b)

\section{2.4 Alternative methods to Kalman condition for regional controllability of CA}

The notion of controllability was identified by Kalman as one of the central properties determining system behaviour. His simple rank condition is prevalent in the analysis of linear systems. In this section, we aim at exploring new approaches to prove the regional controllability of $\mathrm{CA}$ in more general cases.

\subsubsection{Markov Chains approach for controllability}

The first method comes from the idea that CA and Markov-Chain modelling are of great interest when merged and applied in practical situations. The evolution of all possible configurations of a probabilistic CA can be written as a Markov chain. Since deterministic CA are limit cases of probabilistic ones, they also can be seen as particular Markov chains. A Markov chain such that, for some $t, M^{j}>0$ for all $i, j$ is said to be regular, and this implies that any configuration can be reached by any configuration in time $t$. The evolution of a controlled CA can be seen as a Markov chain where the states are the possible configurations of region $\omega$. Two CA configurations restricted to the target region $\omega,\left.s_{0}\right|_{\omega}$ and $\left.s_{1}\right|_{\omega}$, are related to each other if it exists a boundary control $(l, r)$ such that $p_{s_{0}, s_{1}}=1$, where $p_{s_{0}, s_{1}}$ denotes the probability for jumping from $s_{0}$ to $s_{1}$ in one step. In that way, the so-called transition matrix can be constructed in order to describe and analyse a Markov Chain.

Example 2. Consider the CA rule 150 and its transition matrix and associated graph, as shown in Fig. 1. 


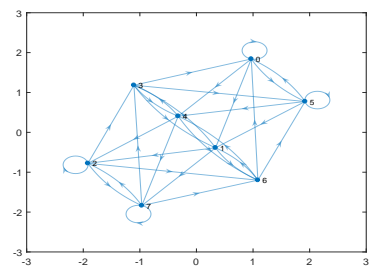

Figure 1: (left) The transition matrix $\mathcal{C}_{150}$ for $|\omega|=3$. (right) Graph of $\mathcal{C}_{150}$.

Theorem 3. A Cellular Automata (linear or non linear) is regionally controllable if and only if it exists a power of matrix $\mathcal{C}^{T}$ such as all the components are strictly positive.

\subsubsection{Graph theory approach for regional controllability of CA}

Markov chains can be described using directed graphs where the nodes represent the different possible states and the edges represent the probability of the system moving from one state to the other in the next time instance. The graph theory seems to be a good and appropriate tool to study the problem of regional controllability of CA. We give the important characterisation result in terms of graph Theory.

Theorem 4. A Cellular Automata is regionally controllable iff there exists a $t$ such that the graph associated to the transformation matrix $\mathcal{C}^{t}$ contains a Hamiltonian circuit, that is a circuit of a graph $G=(V, A R)$ is a simple directed path of $G$ that includes every vertex exactly once [11.

\section{Observability and Reconstructibility of Cellular Automata}

\section{3.1 Introduction to Observability and Reconstructibility}

Observability, as defined by Kalman [20, 21], allows to determine if a system is observable by one or more sensors, i.e. if it is possible to reconstruct the state of the system based on the measurements of these sensors. This criterion makes it possible to choose and place efficiently the different sensors needed for the observation. Once the sensors are placed so that they can observe the system, it is necessary to build the observer, an entity that estimates the state of the system from the measurements of the sensors. As the observability criterion is verified, we know that such an observer exists.

The estimated state of the system, noted $\hat{z}$, is constructed from the $y$ measurements taken by the sensors and from the dynamics of the physical system. Its mathematical description is similar to the augmented definition (112) of the physical system except for the addition 
of an output error feedback via the observer operator $L$. The estimated state dynamics satisfy therefore

$$
\left\{\begin{array}{l}
\dot{\hat{z}}(t)=A \hat{z}(t)+L(y(t)-\hat{y}(t))+B u(t) ; 0<t<T \\
\hat{y}(t)=C \hat{z}(t) \\
\hat{z}(0)=\hat{z}_{0} \in D(A)
\end{array}\right.
$$

The $L$ operator represents the process by which the estimated state converges towards the state of the system; the way it is constructed influences the performance or the robustness of the observer.

In most studies, observability, and by extension all the aspects related to observation, are studied through controllability by the principle of duality that binds one to the other [20]. In this section, we will pay particular attention to the use of observation for monitoring and estimation of initial state for complex systems modelled by cellular automata, specifically autonomous cellular automata, i.e. those which are not controlled $(\forall t \in[0, T], u(t)=0)$. In the following we present separately our study on CA monitoring and CA initial state estimation. The first one consists in determining the current state of the system while the second one focuses on finding out the initial state. These two notions are called respectively reconstructibility and observability [17. The latter is stronger than the former because if the initial state can be estimated, then it is possible to compute the current state from this initial state. Reconstructibility is rarely addressed in the study of linear continuous-time invariant (LTI) systems or continuoustime DPS because of its equivalence to observability (which is not anymore valid for discrete-time systems, see [1). It is however starting to be investigated in the case of the Boolean Control Network [17, 41].

\section{3.2 Observability and Reconstructibility of CA}

Cellular automata are mathematical models where time, space and state are discrete. This total discretization prevents the direct use of the observability and controllability results of continuous time control theory, or even discrete time only control theory. Indeed, the existence and uniqueness of an inverse requires the state space to be a field (in the mathematical sense). To build a finite (or Galois) field from only a finite number of possible state values, requires this number to be prime. On the other hand, the finiteness of the state values makes it possible to use discrete mathematics results such as, for instance, those from graph theory. The behaviour of the system can be represented in the form of a graph, called configuration graph, that links the different possible states of the system [29. This discrete approach to observability and controllability is recent in control theory, but not in computer science nor in applied mathematics. There are numerous 
methods of state reconstruction but they are not based on observability and reconstructibility criteria.

From the definition presented in the section 2.2 .2 of the global dynamics $(F)$ and observation $(H)$ operators, a sequence of output $\Theta$ around a time horizon $T$ can be reconstructed from the initial state, through some extended observability (or gramian) operator:

$$
\Theta_{T}: s_{0} \mapsto\left\{\theta_{0}, \theta_{1}, \ldots, \theta_{T-1}\right\}=\left\{H s_{0}, H F s_{0}, \ldots, H F^{T-1} s_{0}\right\}
$$

where $\Theta_{T}$ represents the $T$ first outputs of the initial configuration $s_{0} \in X$. Plenet et al. 29] proposed a definition for the observability and reconstructibility that can both be mathematically linked to the property of injectivity of the the output sequence $\Theta_{T}$.

Definition 4 (Observability \& Reconstructibility) Let $A$ be a cellular automata with a global transition function $F$ and an output sequence $\Theta_{T}$, then the two following propositions hold:

- $A$ is observable $\Longleftrightarrow \forall s_{0}^{\prime}, s_{0}^{\prime \prime} \in X, \Theta_{T}\left(s_{0}^{\prime}\right)=\Theta_{T}\left(s_{0}^{\prime \prime}\right) \Longrightarrow s_{0}^{\prime}=s_{0}^{\prime \prime}$

- $A$ is reconstructible $\Longleftrightarrow \forall s_{0}^{\prime}, s_{0}^{\prime \prime} \in X, \Theta_{T}\left(s_{0}^{\prime}\right)=\Theta_{T}\left(s_{0}^{\prime \prime}\right) \Longrightarrow F^{T}\left(s_{0}^{\prime}\right)=$ $F^{T}\left(s_{0}^{\prime \prime}\right)$

From this definition, it is clear that both can be used to reconstruct a configuration from the measurements as every output sequence is the result of only one configuration. Moreover, each proposition deals with a specific configuration: the observability deals with the initial configuration $\left(s_{0}\right)$ whereas the reconstructibility deals with the current configuration $\left(s_{t}=F^{T}\left(s_{0}\right)\right)$. In addition, observability is stronger than reconstructibility as $s_{T}$ can be calculated from $s_{0}$, thus the observability must be assessed before the reconstructibility.

The observability of cellular automata is scarcely studied, even for systems with stationary sensors. The following sections present two methods to determine CA observability: a function-based method and a configuration-based method. In the first one, the algebraic properties of the transition function (local or global) are studied to derive observability properties. In the second one, the discrete aspect of cellular automata is combined with the notion of relationship between the automata configurations to get information about the CA's observability. The function-based method has the advantage of a linear complexity with the number of cells since each cell has a finite number of states. Conversely, the configuration-based method has exponential complexity since the number of configuration depends on the number of cells and the number of states (for instance, a Boolean cellular automata with $N$ cells has $2^{N}$ possible configurations).

Complex Systems, Volume (year) 1-1+ 


\subsubsection{Observability of CA: Kalman Condition}

This section present a function-based method to determine observability for affine cellular automata through the affine property of the transition function. It relies on the Kalman condition presented in theorem 1, and on the controllability/observability duality. A cellular automata is said to be affine if its $F$ transition function is affine, i.e. it uses only addition and multiplication by constants. The transition function can then be expressed with a linear map and a constant vector.

Definition 5 (Affine Cellular Automata) If a cellular automata and an output operator are affine then $F$ (respectively $H$ ) can be written in the form of a linear map plus a constant, which can in turn be written as a matrix $A$ and a constant $\eta=F(0)$ (respectively $C$ and $\gamma=H(0)$ ). The evolution of the cellular automata can therefore be written as:

$$
\begin{cases}s_{t+1} & =F\left(s_{t}\right)=A s_{t}+\eta \\ \theta_{t} & =C s_{t}+\gamma \\ s_{0} & \in X\end{cases}
$$

Definition 6. The output sequence $\Theta_{T}$ is also an affine map composed with $O_{T}$ the observability matrix (the dual of the controllability matrix $M_{c}$ presented in def 2 and $\Gamma_{T}$ a constant vector.

$$
\Theta_{T}=\left[\begin{array}{c}
\theta_{0} \\
\theta_{1} \\
\ldots \\
\theta_{T-1}
\end{array}\right]=\underbrace{\left[\begin{array}{c}
C 0 \\
C A \\
\ldots \\
C A^{T-1}
\end{array}\right]}_{O_{T}} s_{0}+\underbrace{\left[\begin{array}{c}
\gamma \\
C J_{0} \eta+\gamma \\
\ldots \\
C J_{T-2} \eta+\gamma
\end{array}\right]}_{\Gamma_{T}}=O_{T} s_{0}+\Gamma_{T}
$$

with $J_{t}=\sum_{k=0}^{t} A^{k}$

The Kalman rank condition for the observability of an affine CA is the following:

Theorem 5 (Kalman Rank Condition) An affine cellular automata $F$, observed by an affine output operator $H$, is observable under a time horizon $T$, if and only if $O_{T}$ is full rank

This Kalman rank condition is usually used for continuous and discrete LTI systems and was extended to affine cellular automata [28, 29]. A corollary is also given allowing the reconstruction of the initial state from the measurements by inverting (or pseudo inverting) the full rank matrix $O_{T}$.

Corollary 1. If the Kalman criterion is verified, then it is possible to recover the initial state by inverting the observability matrix:

$$
x_{0}=O_{T}^{\dagger}\left(Y_{T}-\Gamma_{T}\right)
$$


This theorem and corollary are applicable for an observable system but can be extended in the case of a reconstructible system with the following theorem and corollary.

Theorem 6 (Reconstructibility Condition) An affine cellular automata $F$, observed by an affine output operator $H$ is reconstructible if and only if there exists a finite time horizon $T$ such that:

$$
\operatorname{ker} O_{T} \subset \operatorname{ker} A^{T}
$$

Corollary 2. If the reconstructibility criterion is verified, then it is possible to find a matrix $R$ such that:

$$
x_{T}=R\left(Y_{T}-\Gamma\right)+J_{T-1} \eta
$$

These two theorems and corollaries allow to assess the observability and reconstructibility only for affine cellular automata. This class of automata is in fact quite small. For instance, among the Wolfram's 256 elementary CA, only 16 are affine: the 8 additive rules $(0,60,90$, $102,150,170,204,240)$ and their complementary rules $(255,195,165$, $153,105,85,51,15)$. Therefore, a second method has been proposed to investigate observability and reconstructibility of CA control systems. It is presented hereafter.

\subsubsection{Observability of CA: Configuration Relation}

This configuration-based method makes use of a binary relation representation of the $F, H$ and, $\Theta$ operators, respectively noted $\mathcal{R}_{F}, \mathcal{R}_{H}$, and $\mathcal{R}_{\Theta}$.

Definition 7. The transition binary relation $\mathcal{R}_{F}$ associated to the global transition function $F$ is defined as follows:

$$
\mathcal{R}_{F}=\left\{\left(s_{0}, s_{1}\right) \mid s_{0}, s_{1} \in X \text { and } s_{1}=F\left(s_{0}\right)\right\}
$$

The binary relation can be represented in the form of a matrix called logical matrix.

Definition 8. The logical matrix $M$ associated to a binary relation $\mathcal{R} \in X \times Y$ is defined as:

$$
M_{i, j}= \begin{cases}1 & \left(x_{i}, y_{j}\right) \in \mathcal{R} \\ 0 & \text { else }\end{cases}
$$

This binary relation representation allows to assess the observability and reconstructibility of all kinds of cellular automata, independently of their algebraic nature. From the definition 4, it is clear that the observability and the reconstructibility is related to the injectivity of 
$\Theta_{T}$. This property can be studied through the relation $\mathcal{R}_{\Theta}$ for the observability and $\mathcal{R}_{\Theta_{T} F^{-T}}$ (constructed from $\mathcal{R}_{\Theta}$ and the converse of $\mathcal{R}_{F^{T}}$ ) for the reconstructibility. Which results in the following theorem using the logical matrices of such relations:

Theorem 7. A cellular automata is observable (reconstructible) if the logical matrix related to the binary relation $\mathcal{R}_{\Theta}\left(\mathcal{R}_{\Theta_{T} F^{-T}}\right)$ has at most one non-zero element per column.

These logical matrices have very few non-zero elements, making it possible to use sparse matrices to represent them numerically. This reduces not only the memory allocation but also the algorithmic complexity for the assessment of observability and reconstructibility.

The main problem with this method is the exponential increase in the number of configurations in relation with the number of cells. Even when the algorithms used to evaluate observability are optimised, the exponential complexity coming from the number of configurations makes this method less efficient when the particular case of affine cellular automata is investigated.

\section{3.3 Observation of DPS through Mobile Sensors}

In the case of "classic" observation problems, only the sensors types are chosen accordingly to the natures of the output variables to be measured and the state variables to be observed (e.g. a displacement, velocity or force for a mechanical system). But for spatially distributed systems, the position of the sensors becomes as well a crucial problem in the design of the observer. The placement of sensors remains, even today, a major concern in many areas: water networks, forest fire detection, tele-monitoring of human physiological data, etc. Sensor placement is often related to the wireless sensor network (WSN) research area [39, 38, which studies strategies to place sensors to get optimal information about an observed system. The main difference with our approach, based on control theory and observation, is that WSNs do not seek to reconstruct the state of the system using its dynamics. Sensor placement is carried out using different algorithmic methods: genetic algorithms [37, Bayesian optimisation [18, etc. In these studies, the sensors are generally considered fixed. This reduces in fact their performance for the observation of spatially distributed systems. Indeed, the use of mobile sensors makes it possible to obtain observability whereas stationary sensors would have failed [29]. However, this poses new problems such as the calculation of sensor trajectories, which is still under investigation. Some researchers are directly studying the problem of observability with mobile sensors. Noticeably Demetriou et al [6, 7] or Hussein et al [19] are considering networks of mobile actuators and/or sensors. Related problems occurred when mobile sensors 
(or actuators) are considered such as obstacle avoidance or formation coordination to get maximum coverage. Such problems are intensively investigated, independently from the observation observation problem. For instance, among the many different existing methods to handle collision avoidance for the mobile sensors, one may cite the predictive control approach [27] or the potential field approach [24.

As the sensors are moving in space, the cell they observe change through time. The output operator $C$ becomes therefore time-dependent (it may then be denoted $C(t)$ ). The observability criteria become timedependent and it is therefore necessary to evaluate the observability for all the different sensor trajectories. The two observability conditions presented in the section 3.2 can both be assessed with mobile sensors but for only one trajectory at a time. They can be used as a constraint in constrained trajectory planning to find a mobile sensor trajectory that respects observability and other constraints external to the observability problem such as obstacle avoidance. This trajectory planning can be carried out either prior to system deployment or in real time.

\section{Discussion}

Controllability and observability are two fundamental properties that characterise the behaviour of a given system and determine the relationships between state, input and output variables. As the real physical systems become more and more complex, the need to design controllers and observers for complex nonlinear systems is real. The study of controllability of linear systems was first performed in detail by Kalman and his collaborators in [21. The first part of this paper discussed an analogue of the Kalman controllability rank condition for systems described by cellular automata considered as the discrete counterpart of partial differential equation. It also explored some alternative approaches to this condition that could work for linear and non linear CA. It focuses on the special case of regional controllability of Boolean 1D-CA. A necessary and sufficient condition for regional controllability of CA was proved through original approaches such that Markov Chains and graph theory.

This work can be extended in many tracks that can explore particularly the case of non-Boolean CAs and the high nonlinear CA. The case of large lattice size is also to be considered. The examples considered concerned only a very limited number of cells because of the enormous computational cost generated in terms of spatial and temporal complexity. The control obtained is generally not unique at this stage and the problem of optimality will be addressed later. A first problem of regional controllability in minimum time is currently under study.

Another perspective concerns the investigation of more adequate algorithms for the calculation of preimages that could be of low com-

Complex Systems, Volume (year) 1-1+ 
plexity depending on the dimensions of the lattice.

For the observability issue, two methods for assessing observability and reconstructibility were presented. The first one using the Kalman condition only applies to affine CAs while the second one allows to study non-linear CAs but its exponential complexity makes it difficult to use. Several approaches can be considered to reduce the algorithmic complexity linked to the calculation of observability and reconstructibility. The first one would be a new function-based method but for non-linear CA. Previous strategies already used in the case of LTI systems such as the use of linearization or the use of non-linear Jacobian could be considered. The second method to make observability and reconstructibility computation tractable on CA with large number of cells is to use multiple observers. Each of these smaller and simpler observers is then in charge of the observation of a part of the whole $\mathrm{CA}$. The limited number of possible configurations for each observed region of the CA implies a dimensional reduction making it tractable to determine observability, or reconstructibility on these parts of the CA. For example, with a Boolean controller of 1000 cells, there are $2^{1000}$ configurations. If this automata is observed by 10 observers of 100 cells, then one must evaluate 10 times the observability for $2^{100}$ configurations. However, a new notion of observability will still have to be defined to account for these distributed observers in order to ensure that the local observability of the local observers can be combined to obtain the observability of the whole system.

The use of a mobile sensor network not only ensures observability where static sensors would have failed, but also makes the topology of the observation network dynamic and allows the sensors to focus on a specific part of the system. However, the use of mobile sensors coupled with the distributed observers requires strong coordination of the sensors. These two criteria make it extremely complex to calculate trajectories under constraints using the classical methods of control theory. One approach for improvement would be to use a notion already well studied in computer science: the multi-agent systems (MAS) paradigm. The agents (here observers and sensors) are autonomous and communicate with each other. Each agent would calculate its own trajectory according to the constraints (observability and other) and the information exchanged between the agents. In the same way that the distributed observer manages to reduce the complexity of the observability calculation by distributing the task between several entities, the use of MAS produces the same effect but with respect to the trajectory calculation. The organisation of the network and the communications between agents will have to be carefully designed to ensure global observability of the system based on local computations by the agents.

It should be noted that the controllability and observability prob- 
lems were considered separately in this paper. For controllability issues, we assumed that the state of the system can be measured at each time and for observability problem, the system is supposed to be autonomuous. The global objective of considering a complete state equation with control and observation will be investigated in a future work.

\section{References}

[1] A. C. Antoulas, Approximation of large-scale dynamical systems. Society for Industrial and Applied Mathematics, 2005.

[2] F. Bagnoli, S. El Yacoubi, and R. Rechtman, "Toward a boundary regional control problem for boolean cellular automata", Natural Computing, 17(3), 2018 pp. 479-486, .

[3] B. Chopard and M. Droz, Cellular Automata Modeling of Physical Systems, Cambridge University Press, 1998.

[4] RF. Curtain and H. Zwart, An introduction to infinite dimensional linear systems theory. Springer, Heidelberg, 1995.

[5] C. Damiani, R. Serra, M. Villani, S.A. Kauffman, and A. Colacci, "Cellcell interaction and diversity of emergent behaviours". IET systems biology, 5(2), 2011 pp. 137-144.

[6] M. A. Demetriou, "Guidance of mobile actuator-plus-sensor networks for improved control and estimation of distributed parameter systems". IEEE Transactions on Automatic Control, 55(7), 2010 pp. 1570-1584.

[7] M. A. Demetriou and I. I. Hussein, "Estimation of spatially distributed processes using mobile spatially distributed sensor network", SIAM Journal on Control and Optimization, 48(1), 2009 pp. 266-291.

[8] A. Deutsch and S. Dormann, Cellular Automaton Modeling of Biological Pattern Formation Characterization, Examples, and Analysis, Birkhũser Boston. 2005

[9] S. Dridi, S. El Yacoubi, and F. Bagnoli, "Boundary regional controllability of linear boolean cellular automata using markov chain", Recent Advances in Modeling, Analysis and Systems Control: Theoretical Aspects and Applications, 243. Springer, Cham, 2020 pp. 37-48 (2020).

[10] S. Dridi, F. Bagnoli, and S. EL Yacoubi, "Markov chains approach for regional controllability of deterministic cellular automata, via boundary actions", Journal of Cellular Automata 14(5/6), 2019 pp. 479-498.

[11] S. Dridi, S. El Yacoubi, F. Bagnoli, and A. Fontaine, "A graph theory approach for regional controllability of boolean cellular automata", International Journal of Parallel, Emergent and Distributed Systems, 2019 pp. 1-15. https://doi.org/10.1080/17445760.2019.1608442.

Complex Systems, Volume (year) 1-1+ 
[12] A. El Jai and M. Amouroux, "Sensors and observers in distributed parameter systems", International Journal of Control, 47(1), 1988 pp. $333-347$.

[13] A. El Jai, "Distributed systems analysis via sensors and actuators", Sensors and Actuators A: Physical 29(1), 1991 pp. 1-11.

[14] A. El Jai and S. El Yacoubi, "On the relations between actuator structures and final constraint minimum-energy problem". Sensors and Actuators A: Physical 33(3), 1992 pp. 175-182.

[15] A. Elnagar and A. Hussein, "On optimal constrained trajectory planning in 3D environments". Robotics and Autonomous systems, 33(4), 2000 pp. $195-206$.

[16] G.B. Ermentrout and L. Edelstein-Keshet, "Cellular automata approaches to biological modeling". Journal of theoretical Biology, 160(1), 1993 pp. $97-133$.

[17] E. Fornasini and M. E. Valcher, "Observability, reconstructibility and state observers of Boolean control networks". IEEE Transactions on Automatic Control, 58(6), 2012 pp. 1390-1401.

[18] R. Garnett, M. A. Osborne, and S. J. Roberts, "Bayesian optimization for sensor set selection", in Proceedings of the 9th ACM/IEEE international conference on information processing in sensor networks $2010 \mathrm{pp}$. 209-219.

[19] I. I. Hussein and D. M. Stipanovic, "Effective coverage control for mobile sensor networks with guaranteed collision avoidance". IEEE Transactions on Control Systems Technology, 15(4), 2007 pp. 642-657.

[20] R.E. Kalman, "On the general theory of control systems", in Proceedings First International Conference on Automatic Control, Moscow, USSR, 1960 .

[21] R.E. Kalman, "Mathematical description of linear dynamical systems", Journal of the Society for Industrial and Applied Mathematics, Series A: Control 1(2), 1963 pp. 152-192.

[22] I. Kaarer, A. Ayadi, and I. Rezzoug, "Weak controllability and the new choice of actuators", Global Journal of Pure and Applied Mathematics, 14(2): 2018 pp. 325-330.

[23] S.A. Kauffman, "Homeostasis and differentiation in random genetic control networks", Nature 224, 177178, 1969.

[24] O. Khatib, "Real-time obstacle avoidance for manipulators and mobile robots", Autonomous robot vehicles, New York, NY: Springer. 1986 pp. 396-404.

[25] L.B. Kier, P.G. Seybold, and C-K. Cheng, Modeling chemical systems using cellular automata, Springer Science \& Business Media, 2005.

Complex Systems, Volume (year) 1-1+ 
[26] J.-L. Lions, "Controlabilité exacte des systèmes distribués: remarques sur la théorie générale et les applications", Analysis and optimization of systems, Springer, 1986 pp. 3-14.

[27] J. M. Park, D. W. Kim, Y. S. Yoon, H. J. Kim, and K. S. Yi, "Obstacle avoidance of autonomous vehicles based on model predictive control", in Proceedings of the Institution of Mechanical Engineers, Part D: Journal of Automobile Engineering, 223(12), 2009 pp. 1499-1516.

[28] T. Plénet, S. El Yacoubi, C. Raïevsky, and L. Lefèvre, "Observability of Affine Cellular Automata through Mobile Sensors", accepted for publication in LNCS, ACRI, 2020.

[29] T. Plénet, S. El Yacoubi, C. Raïevsky, and L. Lefèvre, "Observability and Reconstructibility of Cellular Automaton", submitted for publication in Int. Journal of Systems Science.

[30] D.L. Russell, "Controllability and stabilizability theory for linear partial differential equations: recent progress and open questions", Siam Review, 20(4): 1978 pp. 639-739.

[31] G.Y. Vichniac, "Boolean derivatives on cellular automata", Physica Nonlinear Phenomena, 45(1-3): 1990 pp. 63-74.

[32] S. Wolfram, Cellular Automata and Complexity, Addison-Wesley, Science Mathematics Computing, 1994.

[33] A. Wuensche and M. Lesser, "The global dynamics of cellular automata", In An Atlas of Basin of Attraction Fields of One-Dimensional Cellular Automata, Addison-Wesley, 1992.

[34] X. Xiao, S. Shao, Y. Ding, Z. Huang, X. Chen, and K.-C. Chou, "Using cellular automata to generate image representation for biological sequences", Amino acids, 28(1), 2005 pp. 29-35.

[35] S. El Yacoubi, A. El Jai, and N. Ammor, "Regional controllability with cellular automata models", Lecture Notes on Computer Sciences, 14(3), 2002 pp. 227-253.

[36] S. El Yacoubi, "A mathematical method for control problems on cellular automata models", International Journal of Systems Science, 5(39), 2008, pp. 529-538.

[37] L. Yao, W. A. Sethares, and D. C. Kammer, "Sensor placement for onorbit modal identification via a genetic algorithm", AIAA journal, 31(10), 1993 pp. $1922-1928$.

[38] J. Yick, B. Mukherjee, and D. Ghosal, "Wireless sensor network survey", Computer networks, 52(12), 2008 pp. 2292-2330.

[39] M. Younis, and K. Akkaya, "Strategies and techniques for node placement in wireless sensor networks: A survey", Ad Hoc Networks, 6(4), 2008 pp. 621-655.

Complex Systems, Volume (year) 1-1+ 
[40] E. Zerrik, A. Boutoulout, and A. El Jai, "Actuators and regional boundary controllability for parabolic systems", International Journal of System Science, 31, 2000 pp. 73-82.

[41] K. Zhang, L. Zhang, and R. Su, "A weighted pair graph representation for reconstructibility of Boolean control networks", SIAM Journal on Control and Optimization, 54(6), 2016 pp. 3040-3060. 